\title{
Role of Fibronectin on the Clearance and Tissue Uptake of Antigen and Immune Complexes in Rats
}

\author{
F. G. Cosio and A. P. Bakaletz \\ Department of Medicine, The Ohio State University, Columbus, Ohio 43210
}

\section{Abstract}

In the present study, we have evaluated how plasma fibronectin (FN) and tissue FN can affect the clearance from the circulation and organ uptake of antigen or immune complexes (IC) that have the capacity to bind to FN. Phenylated gelatin (DNP-GL) (a FN binding antigen) and IC composed of DNPGL and monoclonal IgGl anti-dinitrophenol (DNP) antibodies were tested. These probes were compared with DNP-bovine serum albumin (BSA) (a non-FN-binding antigen) and DNPBSA IC formed with the same anti-DNP antibody used for the preparation of DNP-GL IC. We found evidence that DNP-GL, but not DNP-BSA, formed complexes with soluble FN in vitro and the data strongly suggest that DNP-GL-FN complexes form in vivo. The formation of complexes with plasma FN aided in the clearance of DNP-GL from the circulation, as shown by the facts that DNP-GL was removed from the circulation much faster than DNP-BSA and that complexes of DNP-GL with plasma FN were removed from the circulation faster than uncomplexed DNP-GL. The sites of deposition of DNP-GL were also different from those of DNP-BSA. Thus, DNP-GL demonstrated higher hepatic, splenic, and renal uptake than did DNP-BSA. Renal uptake of DNP-GL was quite high despite the fact that DNP-GL is anionic. Indeed, expressed per gram of tissue, liver and kidney deposition of DNP-GL was not significantly different. By immunofluorescence microscopy, DNP-GL could be demonstrated in hepatic sinusoids and glomerular mesangium. In vitro, DNP-GL bound to FN in the mesangium of frozen sections of kidney tissue. IC formed with DNP-GL or DNP-BSA demonstrated virtually the same size, yet the fate of DNP-GL IC was strikingly different from that of DNP-BSA IC. The removal of DNP-GL IC from the circulation was mediated by the antigen and not by Fc receptors since gelatin (an inhibitor of DNP-GL clearance) but not aggregated IgG (an inhibitor of Fc receptors) inhibited the removal of DNP-GL IC from the circulation. In summary, these studies suggest that the ability of an antigen or IC to bind to FN markedly influences the fate of that antigen or IC. Specifically, binding to $\mathrm{FN}$ accelerates clearance from the circulation and favors hepatic and renal (primarily mesangial) uptake of the FN binding antigen of IC.

This work was presented in abstract form at the American Federation of Clinical Research, Chicago, IL, on November 1986.

Send all correspondence and reprint requests to Dr. F. G. Cosio, The Ohio State University, N-210 Means Hall, 1654 Upham Drive, Columbus, $\mathrm{OH} 43210$.

Received for publication 8 December 1986 and in revised form 11 June 1987.

J. Clin. Invest.

(c) The American Society for Clinical Investigation, Inc. $0021-9738 / 87 / 11 / 1270 / 10 \quad \$ 2.00$

Volume 80, November 1987, 1270-1279

\section{Introduction}

Most cases of glomerulonephritis are caused by deposition or formation of immune complexes (IC) ${ }^{1}$ in the glomeruli. However, the factors that determine IC deposition or formation in tissues are only partially understood. IC can be formed in situ in glomerular structures when antigen and antibody deposit sequentially (1). Also, IC may form in the circulation and subsequently deposit in glomeruli $(2,3)$. Regarding this later mechanism, it has been shown that the injection of preformed IC into the circulation of experimental animals results in glomerular deposition of only small amounts of IC. The fact that preformed IC do not deposit readily in normal glomeruli has lead to the suggestion that to deposit large amounts of IC in glomeruli, either the glomerulus must be altered to enhance IC uptake or the IC must have special characteristics that foster deposition in glomeruli. Indeed, certain IC-related factors, such as antigen or IC charge, have been shown to facilitate the binding of cationic antigens or IC to negatively charged glomerular structures (4). Also, compounds with capacity to bind to glycoproteins bind to glomerular structures after injection into the renal circulation (5).

In this present study we evaluated the possibility that the capacity of an antigen or an IC to bind the specific glomerular protein fibronectin (FN) may also be an important determinant of glomerular accumulation of these probes. Our interest in FN as a glomerular "acceptor" for antigens and IC is based on several properties of this glycoprotein and on the knowledge that several antigens that participate in human glomerulonephritis have the capacity to bind to $\mathrm{FN}$ in vitro. Those antigens include DNA (6) and antigens derived from bacteria such as Staphylococcus aureus, Streptococcus viridans, and group A streptococcus $(7,8)$, bacteria known to be associated with IC mediated glomerulonephritis. Furthermore, it has been demonstrated that, in vitro, DNA binds to glomerular basement membranes (9), and that the binding is mediated by FN (10).

FN is a large glycoprotein normally present in plasma and tissues. In the kidney, FN is mainly present in the glomerular mesangium and in subendothelial spaces (11). Thus, glomerular FN may serve as a glomerular acceptor for FN binding antigens and IC. Also, several lines of evidence suggest that plasma FN may have an important role by participating in the removal of FN binding compounds from the circulation. For example, specific membrane receptors for FN have been demonstrated in cells of the mononuclear phagocytic system (MPS) $(12,13)$. MPS cells exposed to FN demonstrate an

1. Abbreviations used in this paper: AIgG, aggregated IgG; ANOVA, analysis of variance; DNP, dinitrophenol; DNP-BSA, phenylated bovine serum albumin; DNP-GL, phenylated gelatin; FN, fibronectin; GL, gelatin; IC, immune complexes; ID, injected dose; IF, immunofluorescence; MPS, mononuclear phagocytic system; $N$, No. of experiments. 
enhanced capacity to bind and internalize particles via $\mathrm{Fc}$ and complement receptors $(14,15)$. FN also appears to serve as a recognition molecule for compounds that by themselves are not recognized by MPS cells. For example, in vitro, FN permits the uptake of gelatin (GL)-coated particles by macrophages (16), and in vivo FN is an opsonin for fibrinogen-degradation products (17) and for collagen-degradation products released into the circulation during major body burns (18).

In this study, we evaluated the role of plasma and tissue FN in the removal from the circulation and tissue uptake of an antigen and of an IC with FN binding capacity. The model chosen for study utilizes GL as the antigen since the binding of GL to FN (19) and the effects of FN on the uptake of GL by macrophages have been well characterized (16).

\section{Methods}

Preparation and characterization of antigens and IC. The antigens used throughout the present study included GL (Sigma Chemical Co., St. Louis, MO), phenylated GL (DNP-GL), and phenylated bovine serum albumin (DNP-BSA) (Sigma Chemical Co.). GL and bovine serum albumin (BSA) were phenylated and the ratio of dinitrophenol (DNP) to protein was calculated as described (20). The preparations of DNP-GL and DNP-BSA used throughout these studies had an average phenylation ratio of 10 DNP groups per GL molecule and 9 DNP groups per BSA molecule. DNP-BSA and DNP-GL were radiolabeled with ${ }^{125}$ I by the chloramine $T$ method.

IgGl monoclonal anti-DNP antibodies were prepared and characterized in our laboratory by methods described previously (20). Antibodies were purified by caprylic acid-ammonium sulfate precipitation (21). Anti-DNP antibodies were radiolabeled by the chloramine T method. For the production of IC, DNP-GL or DNP-BSA were incubated with variable concentrations of IgGl anti-DNP antibodies at $37^{\circ} \mathrm{C}$ for $60 \mathrm{~min}$ and $4^{\circ} \mathrm{C}$ overnight. Before use, IC preparations were centrifuged at $1,000 \mathrm{~g}$ for $10 \mathrm{~min}$ to remove precipitable IC. The size of DNP-BSA, DNP-GL and soluble IC were calculated by centrifugation on isokinetic sucrose gradients (22). The isoelectric point of DNP-GL was calculated by chromatofocusing (20).

In vitro binding of antigens and IC to $F N$. Human and rat $F N$ were purified by affinity chromatography on Sepharose 4B GL columns and characterized as described previously (15). To test the capacity of antigens and soluble IC to bind to soluble FN, radiolabeled DNP-GL, DNP-BSA, or IC were incubated at $37^{\circ} \mathrm{C}$ for $15-60$ min with purified preparations of FN or citrated plasma. At the end of the incubation, the mixtures were fractionated by centrifugation in isokinetic sucrose gradients. 26 fractions were collected from the bottom of the gradient and counted for radioactivity in a gamma scintillation counter (Packard Instrument Co., Inc., Downers Grove, IL).

In vivo experiments. Sprague-Dawley rats (150-200 g) were used. The animals were anesthetized with ether. The probe to be tested was injected intravenous via the tail vein. To calculate the amount of radioactivity injected, the syringes containing the sample to be injected were counted for radioactivity before and after the injection had been completed. The difference was taken as the amount injected (injected dose [ID]). In most experiments $10^{6} \mathrm{cpm}$ were injected per animal. This amount of radioactivity corresponded to 50-100 $\mu \mathrm{g}$ of protein. After the injection was completed, serial blood samples were obtained by tail clipping. To calculate the percent of the total ${ }^{125}$ I that represented protein bound radioactivity, blood samples from rats injected with GL or DNP-GL were incubated with saturated ammonium sulfate $\left(85 \%\right.$ final concentration) at $4^{\circ} \mathrm{C}$ overnight and centrifuged at 2,500 $\mathrm{g}$ for $15 \mathrm{~min}$. Blood samples from rats injected with DNP-BSA were mixed with $10 \%$ TCA and then processed as above. The total radioactivity and the radioactivity in the precipitate were counted and results were expressed as percent precipitable (protein bound) ${ }^{125} \mathrm{I}$. Based on the ID, the radioactivity in the blood was expressed as percent of ID per milliliter of blood. In other experiments (clearance experiments), the radioactivity present in the first blood sample obtained ( 2 min after injection) was considered as $100 \%$, and the rest of the time points were expressed as a percent of that initial point.

At different time points after the injection, animals were killed and the major organs were excised, weighed, and counted for radioactivity. More than $75 \%$ of the ID could be accounted for by the radioactivity contained in blood, liver, spleen, kidney, and lung. Most experiments were completed by $30 \mathrm{~min}$ after the injection and little urine was produced during this time period. However, urine was also counted for radioactivity. To calculate the total amount of the probe deposited in a given tissue specimen, the radioactivity passively retained in the vascular space was subtracted from the total radioactivity in the specimen (see later). The radioactivity in organs was then expressed as percent of ID per gram of tissue. In all experiments, the tail of the animal was counted for radioactivity to assess the completeness of the intravenous injection of the probe. Animals demonstrating $>1 \%$ of the ID in the tail were excluded from the analysis.

The calculation of radioactivity deposited in a given organ included correction for the probe passively retained in the vascular space. The blood content of the organ was calculated in parallel experiments (No. of experiments $[\mathrm{N}]=5$ ) in which rats were injected with ${ }^{125} \mathrm{I}$ rat IgG or with ${ }^{125} \mathrm{I}=\mathrm{IgG}$ and equivalent doses of the nonradiolabeled probe being tested. The purpose of these experiments was to assess the effect, if any, of the injected probe on the blood content of the organ studied. We found that the probes used in this study did not affect the blood volume of the organs studied.

To calculate the removal rate of a specific probe from blood over a given period of time, the following formula was used: percent removed $=(A-B) \times 100 / A$, where $A$ designates protein-bound blood radioactivity at the first time point ( $2 \mathrm{~min}$ ) and $B$, protein-bound blood radioactivity at the end of the experiment or at a specific time point (usually $30 \mathrm{~min}$ ) after injection.

To calculate the percent of the probe catabolized over a given period of time, the following formula was used: percent catabolized $=(C-D) \times 100 / C$, where $C$ designates percent of the total radioactivity present in blood as protein-bound radioactivity at $2 \mathrm{~min}$ (first time point) and $D$, percent of the total radioactivity present in blood as protein-bound radioactivity at the end of the experiment or a given time (usually $30 \mathrm{~min}$ ) after injection of the probe.

Liver and kidney were excised at the end of the experiment and examined by light and immunofluorescence (IF) microscopy. For light microscopy, tissues were fixed in $10 \%$ formalin and stained by routine hematoxilyn eosin. For IF microscopy, tissues were frozen at $-70^{\circ} \mathrm{C}$ and $4 \mu \mathrm{m}$ cryostat sections of tissue were evaluated for the presence of antigen or antibody. The presence of DNP-GL was demonstrated by indirect IF by incubating the tissue first, with mouse monoclonal antiDNP antibody and second, with fluorescein isothiocyanate (FITC)-labeled, rat IgG-absorbed goat anti-mouse IgG (Cappel Laboratories, Cochranville, PA). Antibody deposited in tissues was detected by direct IF microscopy by incubating tissues with FITC-labeled goat antimouse IgG. Tissue IF was evaluated semiquantitatively in an epifluorescence microscope (Carl Zeiss, Inc., Thornwood, NY).

In vitro binding of DNP-GL and DNP-GL IC to kidney. To test the ability of DNP-GL or DNP-GL IC to bind to kidney tissues in vitro, 4 $\mu \mathrm{m}$ cryostat sections of rat kidney were attached to microscopic slides and fixed with acetone. Subsequently, different concentrations of DNP-GL or DNP-GL IC were added to the tissue and incubated at room temperature for $30 \mathrm{~min}$. After extensive washing, the presence of antigen and antibody was detected by indirect and direct IF as described above. Control tissues were incubated only with primary and secondary antibody.

To test the specificity of the binding of DNP-GL or DNP-GL IC to renal tissues, renal slices were incubated with GL before the addition of DNP-GL or DNP-GL IC. In other experiments, DNP-GL or DNP-GL IC were preincubated with soluble purified FN $\left(37^{\circ} \mathrm{C}\right.$ for $\left.30 \mathrm{~min}\right)$ before incubation with the renal slice. In some experiments, renal specimens were incubated with rabbit anti-FN antibodies (15) before 
incubation with DNP-GL. The subsequent staining of antigen and antibody were done as described above.

Data analysis. Results throughout this manuscript are expressed as means \pm SE of the mean. Removal rates and catabolic rates were compared by analysis of variance (ANOVA) with Newman-Keuls multiple comparison test. Tissue deposition of the different probes was compared by ANOVA with repeated measures.

\section{Results}

In vitro characterization of DNP-BSA and DNP-GL. The average phenylation ratio of DNP-GL and DNP-BSA was 10:1 and 9:1, respectively. By chromatofocusing, we demonstrated that DNP-GL is an anionic protein with an isoelectric point of 4.2. The isoelectric point of DNP-BSA was 4.9. In isokinetic sucrose gradients, radiolabeled GL and DNP-GL migrated as a single peak with a sedimentation coefficient of $3.5 \mathrm{~S}$, which in the case of gelatin, a random coil, corresponds to a molecular mass of 80,000 D (23). Radiolabeled DNP-BSA migrated as a single peak with a sedimentation coefficient of $4.5 \mathrm{~S}$ corresponding to a molecular mass of $68,000 \mathrm{D}$.

To evaluate the binding to FN in vitro, radiolabeled DNPGL and DNP-BSA were incubated with $100 \mu \mathrm{l}$ of purified FN $(840 \mu \mathrm{g} / \mathrm{ml})$ or with citrated plasma at $37^{\circ} \mathrm{C}$ for $15 \mathrm{~min}$, and the size distribution of the antigen was evaluated in isokinetic sucrose gradients. After incubation with FN or plasma, there was no significant change in the size distribution of DNP-BSA (data not shown). By contrast, there was a significant displacement of DNP-GL incubated with purified FN or plasma (Fig. 1). These experiments suggest that DNP-BSA does not interact with FN. By contrast, DNP-GL binds to FN in solution.

In vivo clearance of $G L, D N P-G L$, and DNP-BSA. Radiolabeled GL and DNP-GL were cleared rapidly from the circulation of rats with $<10 \%$ of the ID remaining in the circulation 30 min after injection (Fig. 2). By contrast, radiolabeled DNPBSA was cleared from the circulation more slowly with $>80 \%$ of the ID remaining in the circulation $30 \mathrm{~min}$ after injection. The removal rate over $30 \mathrm{~min}$ was $96.6 \pm 0.42 \%(N=6)$ for DNP-GL and $16 \pm 0.57 \%(N=4)$ for DNP-BSA $(P<0.0001)$. Also, DNP-GL was catabolized significantly faster than DNP-

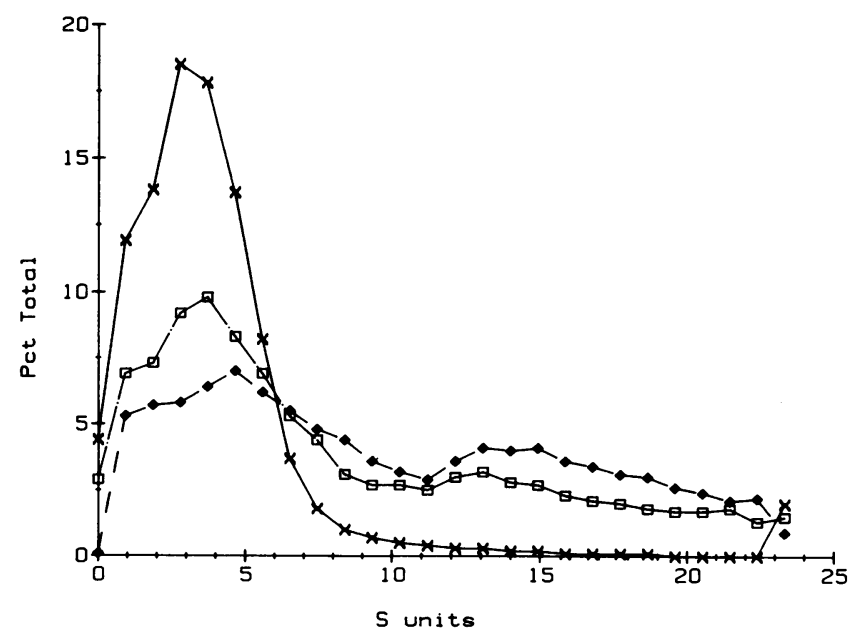

Figure 1. Isokinetic sucrose-gradient fractionation of ${ }^{125}$ I-DNP-GL $(\times),{ }^{125} \mathrm{I}-\mathrm{DNP}-\mathrm{GL}$ preincubated with citrated plasma (ם), and ${ }^{125} \mathrm{I}-$ DNP-GL preincubated with purified FN $(\bullet)$. Values on the $x$-axis represent actual sedimentation coefficients.

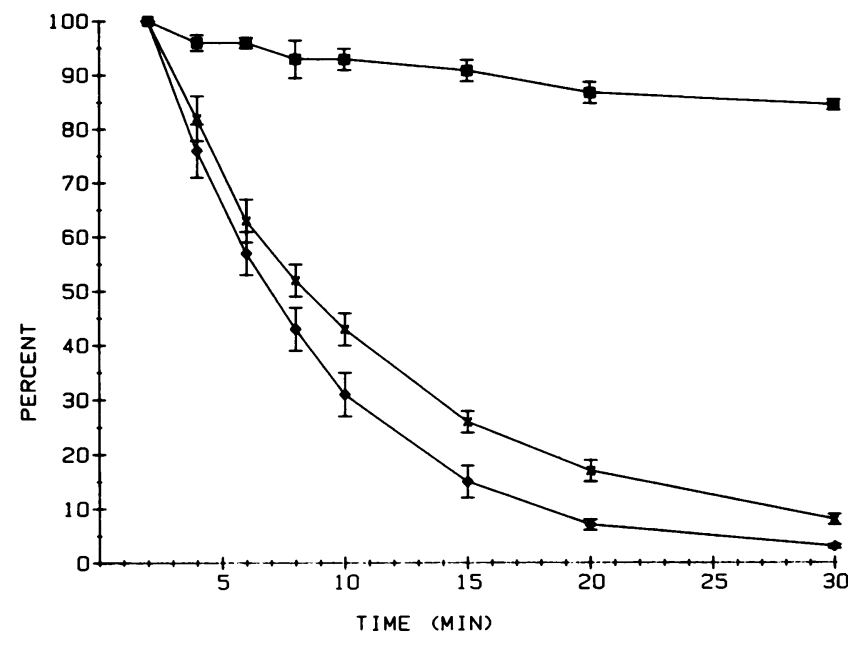

Figure 2. Removal from the circulation of ${ }^{125} \mathrm{I}-\mathrm{GL}(\mathrm{x}),{ }^{125} \mathrm{I}-\mathrm{DNP}-\mathrm{GL}$ $(\bullet)$, and ${ }^{125}$ I-DNP-BSA ( () . The protein-bound radioactivity in the blood sample was expressed as a percent of the two-min sample that was considered as $100 \%$.

BSA. Thus, the percent catabolized over $30 \mathrm{~min}$ was $60 \pm 4.3 \%$ and $4.3 \pm 1.2 \%$ for DNP-GL and DNP-BSA, respectively $(P$ $<0.0001$ ).

As described above, DNP-GL binds to plasma FN in vitro. Thus, we next sought to evaluate the binding of DNP-GL to circulating FN in vivo. ${ }^{125} \mathrm{I}-\mathrm{DNP}-\mathrm{GL}$ was injected into three rats and blood samples collected 5, 10, and 15 min after injection. Citrated plasma was separated by centrifugation and fractionated in isokinetic sucrose gradients (Fig. 3). There was no appreciable binding of ${ }^{125} \mathrm{I}-\mathrm{DNP}-\mathrm{GL}$ to circulating blood cells. 5 min after injection, $69 \pm 5 \%$ of the total ${ }^{125} \mathrm{I}-\mathrm{DNP}-\mathrm{GL}$ in plasma demonstrated higher sedimentation than DNP-GL before injection, indicating that in vivo DNP-GL binds to plasma proteins. This value was determined by calculation of the area under the curve with the use of a polar planimeter (Keuffel \& Esser Co., Morristown, NJ). The size distribution of DNP-GL after in vivo injection was the same as that demonstrated by DNP-GL-FN complexes formed in vitro (see Fig. 1), which suggests that in vivo DNP-GL binds to plasma FN. At 10 and $15 \mathrm{~min}$ after injection, $39 \pm 5 \%$ and $30 \pm 6 \%(N=3)$ of the ${ }^{125}$ I-DNP-GL remained complexed to FN. Compared with the 5-min sample, the 10- and 15-min samples showed a significant decrease in the percent of the total DNP-GL migrating as complexed DNP-GL (Fig. 3). Thus, shortly after injection, DNP-GL binds to plasma FN and DNP-GL-FN complexes appear to be cleared from the circulation faster than free DNP-GL.

To further elucidate the mechanism of clearance of ${ }^{125} \mathrm{I}$ DNP-GL from the circulation, increasing doses of nonradiolabeled DNP-GL were injected into rats together with a constant dose of ${ }^{125}$ I-DNP-GL (Fig. 4). Increasing doses of nonradiolabeled DNP-GL caused progressive decreases in the removal rate of radiolabeled DNP-GL from the circulation. Thus, the mean rates (percent per $30 \mathrm{~min}$ ) were $95.3,73.5,32$, and 25.5 at doses of nonradiolabeled DNP-GL of $0,1,5$, and $10 \mathrm{mg} / \mathrm{kg}$, respectively. By ANOVA, the removal rates in animals injected with 0 or $5 \mathrm{mg} / \mathrm{kg}$ of nonradiolabeled DNP-GL were significantly different $(P<0.0001)$. Also, the percent of the radiolabeled DNP-GL catabolized over $30 \mathrm{~min}$ decreased 


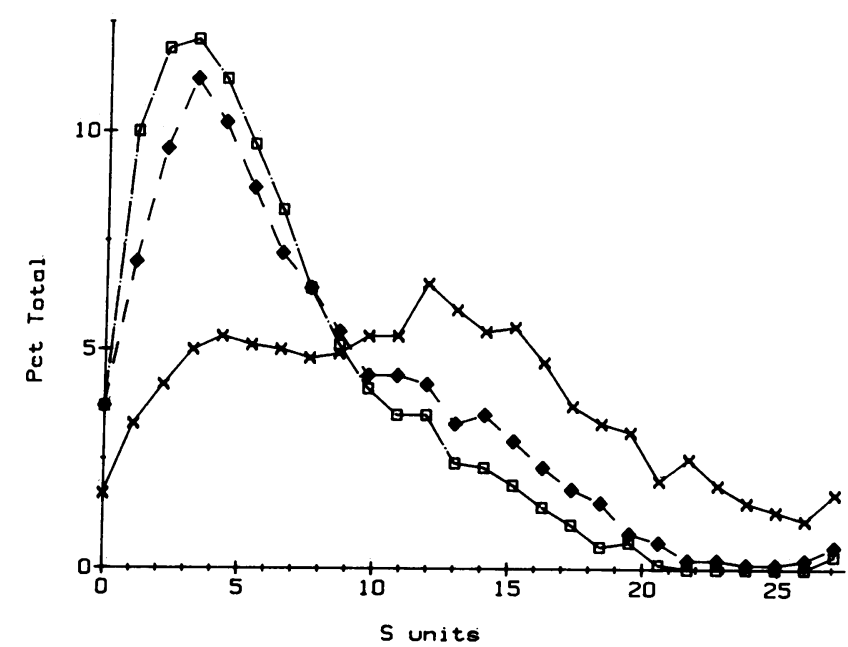

Figure 3. Isokinetic sucrose-gradient fractionation of blood samples obtained from one animal $5(\times), 10(\bullet)$, and $15 \mathrm{~min}(\square)$ after injection of ${ }^{125} I-D N P-G L$. Values on the $x$-axis represent actual sedimentation coefficients.

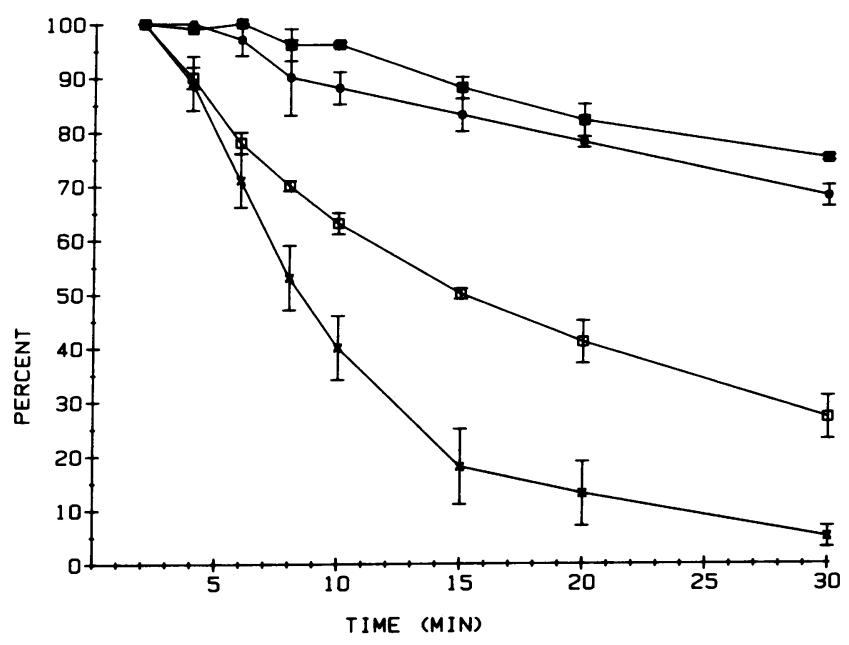

Figure 4. Removal from the circulation of ${ }^{125}$ I-DNP-GL in animals pretreated with DNP-BSA $(10 \mathrm{mg} / \mathrm{kg})(x)$ or DNP-GL at a dose of 1 $\mathrm{gm} / \mathrm{kg}(\square), 5 \mathrm{mg} / \mathrm{kg}(\mathrm{O})$, and $10 \mathrm{mg} / \mathrm{kg}(\square)$. Values represent means $\pm \mathrm{SE}$ and are expressed as a percent of the initial value.

(means of $60,18,3$, and $2 \% / 30 \mathrm{~min}$ ) as the dose of nonradiolabeled DNP-GL was increased $(0,1,5$, and $10 \mathrm{mg} / \mathrm{kg})$. Similar results were obtained in animals pretreated with nonradiolabeled GL instead of nonradiolabeled DNP-GL. By contrast, in four animals pretreated with DNP-BSA $(10 \mathrm{mg} / \mathrm{kg})$, no sig- nificant alteration in the removal from the circulation or catabolism of ${ }^{125} \mathrm{I}-\mathrm{DNP}-\mathrm{GL}$ was observed. Thus, the removal of DNP-GL from the circulation is mediated by a saturable mechanism that is specific for GL.

The inhibition of DNP-GL clearance by increasing doses of GL or DNP-GL could be explained by at least two mechanisms. First, excess nonradiolabeled DNP-GL may saturate the DNP-GL binding capacity of plasma FN. Under those conditions ${ }^{125} \mathrm{I}$-DNP-GL would circulate as uncomplexed antigen that, as we indicated before, is removed from the circulation more slowly than DNP-GL-FN complexes. Second, excess nonradiolabeled DNP-GL may saturate the tissue-binding sites for ${ }^{125}$ I-DNP-GL. To assess which of the above mechanisms is operative, we performed two series of experiments. In the first series, radiolabeled DNP-GL was preincubated with plasma before injection into rats that had been pretreated with an excess nonlabeled DNP-GL ( $1 \mathrm{mg} / \mathrm{kg})$. We reasoned that if binding of ${ }^{125} \mathrm{I}-\mathrm{DNP}-\mathrm{GL}$ to plasma $\mathrm{FN}$ is rate limiting in the removal of the radiolabeled probe from the circulation, then preincubation of the test probe with plasma before injection should increase its removal rate. We found, however, that the removal rate of ${ }^{125} \mathrm{I}-\mathrm{DNP}-\mathrm{GL}$ was not significantly different between animals receiving ${ }^{125} \mathrm{I}-\mathrm{DNP}-\mathrm{GL}$ alone $(70 \pm 3.5 \% / 30$ min, $N=3$ ) and those receiving it preincubated with plasma $(74 \pm 5 \% / 30 \mathrm{~min}, N=3)$. In the second series of experiments, we measured by isokinetic sucrose gradients the percent of the total ${ }^{125}$ I-DNP-GL that formed complexes with plasma FN 10 min after injection into animals pretreated with excess DNPGL $(10 \mathrm{mg} / \mathrm{kg})(N=2)$ or not pretreated $(N=2)$. These experiments demonstrated that the percent of the total ${ }^{125} \mathrm{I}$ DNP-GL complexed to plasma proteins was not significantly different between rats pretreated or not pretreated with excess DNP-GL ( $27 \pm 7 \%$ and $39 \pm 6 \%$, respectively). Thus, excess nonradiolabeled DNP-GL does not significantly inhibit the formation of DNP-GL-FN complexes in the circulation. Together, the results of these series of experiments suggest that increasing doses of DNP-GL saturate the tissue uptake of DNP-GL and that saturation of DNP-GL tissue uptake occurs before the binding capacity of plasma FN for DNP-GL is exceeded.

Tissue uptake of $G L, D N P-G L$, and DNP-BSA. Table I demonstrates the tissue uptake of radiolabeled GL, DNP-GL, and DNP-BSA $30 \mathrm{~min}$ after intravenous injection. By ANOVA with repeated measures a statistically significant effect of probe injected vs. tissue deposition was found (overall $P$ $<0.0001$ ). As can be seen, compared with the tissue uptake of DNP-BSA, both GL and DNP-GL accumulated more in liver, spleen, and kidney. Per gram of tissue, the hepatic and renal uptake of GL and DNP-GL were not different. Thus, the FN binding antigens GL and DNP-GL are avidly taken up by

Table I. Tissue Uptake of Radiolabeled DNP-BSA, GL, and DNP-GL 30 Min after IV Injection

\begin{tabular}{|c|c|c|c|c|c|c|}
\hline Probe & $N$ & Blood & Liver & Spleen & Kidney & Lung \\
\hline DNP-BSA & 3 & $4.2 \pm 0.4^{*}$ & $0.8 \pm 0.1$ & $0.3 \pm 0.03$ & $0.4 \pm 0.11$ & $0.2 \pm 0.1$ \\
\hline GL & 3 & $0.5 \pm 0.3$ & $5.2 \pm 0.2$ & $2.0 \pm 0.3$ & $4.9 \pm 0.3$ & $0.1 \pm 0.03$ \\
\hline DNP-GL & 6 & $0.3 \pm 0.03$ & $5.3 \pm 0.3$ & $1.5 \pm 0.1$ & $5.0 \pm 0.4$ & $0.2 \pm 0.02$ \\
\hline
\end{tabular}

The data are expressed as percent ID per gram of tissue. The percent ID per milliliter of blood present at 30 min is demonstrated for comparison. Tissue uptake was compared by ANOVA with repeated measures. * Results are expressed as means \pm SE. 


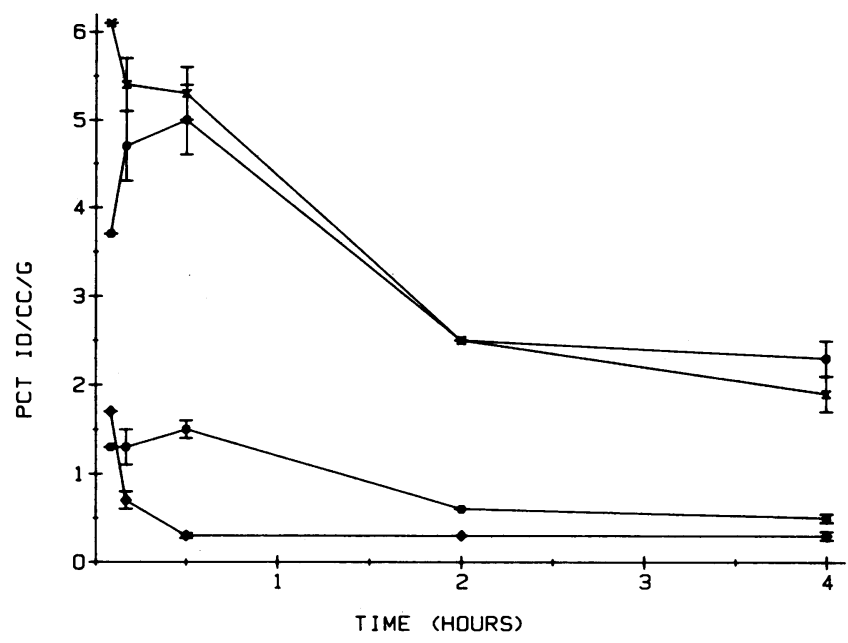

Figure 5. Tissue deposition of ${ }^{125} \mathrm{I}-\mathrm{DNP}-\mathrm{GL}$ at different time points after injection. Tissue counts were corrected for blood contamination of the organ. Liver ( $x$ ); kidney (o), spleen ( $\bullet$ ), and blood $(\bullet)$ counts are represented as the means $\pm \mathrm{SE}(N=3$ at all time points, except at $120 \min N=1$ ).

organs of the MPS, such as the liver and spleen. Also, these antigens are rapidly taken up by the kidney. This is in contrast to DNP-BSA that, as we demonstrated before, has no FN binding properties.

In some experiments, tissue uptake was determined at different times after injection of ${ }^{125} \mathrm{I}-\mathrm{DNP}-\mathrm{GL}$ (Fig. 5). As can be seen, liver uptake occurred rapidly and was maximal at the earliest point $(5 \mathrm{~min})$. Thereafter, hepatic tissue levels declined. Maximal renal uptake of DNP-GL occurred $30 \mathrm{~min}$ after injection and, thereafter, renal tissue levels declined at a rate similar to that of liver. After $24 \mathrm{~h}$, liver, spleen, and kidney tissue levels were $0.6 \pm 0.03,0.2 \pm 0.01$, and $1 \pm 0.06 \% \mathrm{ID} / \mathrm{g}$, respectively.

From the experiments described above (Fig. 4), it was also possible to determine the effect of nonradiolabeled DNP-GL on the tissue uptake of ${ }^{125}$ I-DNP-GL. As can be seen in Table II, increasing doses of nonradiolabeled DNP-GL caused progressive inhibition of the hepatic and splenic uptake of ${ }^{125} \mathrm{I}$ DNP-GL. By contrast, renal uptake of ${ }^{125}$ I-DNP-GL was not significantly inhibited. Indeed, even the lowest dose of nonradiolabeled DNP-GL caused an enhancement in the renal uptake of ${ }^{125}$ I-DNP-GL. Note that the total organ uptake of DNP-GL (labeled and not labeled) reached a maximum of 150 $\mu \mathrm{g} / \mathrm{g}$ of liver and $60 \mu \mathrm{g} / \mathrm{g}$ of spleen in rats injected with $5 \mathrm{mg} / \mathrm{kg}$ of DNP-GL. In kidney, tissue uptake was directly proportional to the total dose of DNP-GL and reached a maximum of 2,560 $\mu \mathrm{g} / \mathrm{g}$ in rats injected with the highest dose of DNP-GL tested $(20 \mathrm{mg} / \mathrm{kg})$. These results suggest that the mechanism of uptake of DNP-GL by MPS organs (liver and spleen) is different from that of kidney. MPS uptake is rapidly saturable, whereas renal uptake of DNP-GL is not saturated by the doses of DNP-GL used in these experiments. Enhanced renal uptake of ${ }^{125}$ I-DNP-GL by excess nonradiolabeled DNP-GL may be related to increased delivery of the probe to the kidney due to higher blood levels. Also, it can be seen that despite the fact that nonradiolabeled DNP-GL inhibits the catabolism of ${ }^{125} \mathrm{I}$ DNP-GL (see above), the renal net uptake of radioactivity is not decreased. This indicates that the high level of renal uptake of radioactivity cannot be attributed to accumulation of nonprotein-bound ${ }^{125} \mathrm{I}$ in the kidney.

To study the anatomic localization of DNP-GL in liver and kidney, animals were injected with $5 \mathrm{mg} / \mathrm{kg}$ of nonradiolabeled DNP-GL and the tissue localization was evaluated by IF microscopy (Fig. 6). Liver localization was evaluated 30 or 60 min after injection and renal localization, in biopsy tissue obtained at $1,2,4,6,24$, and $48 \mathrm{~h}$ after injection. 30 and $60 \mathrm{~min}$ after injection, there was intense staining of hepatic sinusoids for DNP-GL. In the kidney, DNP-GL localized mainly in the glomerular mesangium, although at early time points (up to 4 h) weak interstitial staining was also apparent. Mesangial staining for DNP-GL was persistent up to $24 \mathrm{~h}$ after the injection of antigen, although the intensity of the staining decreased after $6 \mathrm{~h}$ of injection. By light microscopy, no significant abnormalities were demonstrated in liver or kidney tissues.

In vitro binding of DNP-GL to renal tissues. To evaluate the specificity of the binding of DNP-GL to renal tissues, $6 \mu \mathrm{m}$ cryostat sections of kidney were fixed with acetone, incubated with DNP-GL or DNP-BSA $(100 \mu \mathrm{g} / \mathrm{ml})$, and stained by indirect IF as described in the Methods section. In vitro, DNP-GL bound to kidney slices and the localization of the antigen was the same as that seen after in vivo injection, that is, mainly in the glomerular mesangium (Fig. $7 A$ ). Under identical conditions, DNP-BSA did not bind the renal tissues. Preincubation of kidney slices with GL $(10 \mu \mathrm{g} / \mathrm{ml})$ inhibited the subsequent binding of DNP-GL to kidney (Fig. $7 \mathrm{~B}$ ), indicating that this binding is specific for GL and is saturable with concentrations of GL higher than those achieved in vivo. In additional experiments we sought to test the hypothesis that the binding of DNP-GL to the glomerular mesangium is mediated by mes-

Table II. Effects of Increasing Doses of Nonradiolabeled DNP-GL on Tissue Accumulation of ${ }^{125}$ I-DNP-GL 30 Min after Injection into Rats

\begin{tabular}{|c|c|c|c|c|c|c|}
\hline DNP-GL & $N$ & Blood & Liver & Spleen & Kidney & Lung \\
\hline \multicolumn{7}{|l|}{$m g / k g$} \\
\hline 0 & 6 & $0.3 \pm 0.03$ & $5.3 \pm 0.3$ & $1.5 \pm 0.1$ & $5.0 \pm 0.4$ & $0.2 \pm 0.02$ \\
\hline 1 & 1 & 1.0 & 5.6 & 1.8 & 6.2 & 0.2 \\
\hline $5^{*}$ & 3 & $2.6 \pm 0.2$ & $1.5 \pm 0.3$ & $0.6 \pm 0.1$ & $6.1 \pm 0.5$ & $0.1 \pm 0.05$ \\
\hline 10 & 2 & $3.3 \pm 0.1$ & $0.6 \pm 0.01$ & $0.4 \pm 0.01$ & $6.4 \pm 0.5$ & $0.2 \pm 0.01$ \\
\hline 20 & 1 & 4.0 & 0 & 0.04 & 6.4 & 0 \\
\hline
\end{tabular}

Results are expressed as percent of ID per gram of tissue or per milliliter of blood. * Tissue uptake in this group of animals was compared with animals injected with $0 \mathrm{mg} / \mathrm{kg}$ of DNP-GL by ANOVA. 

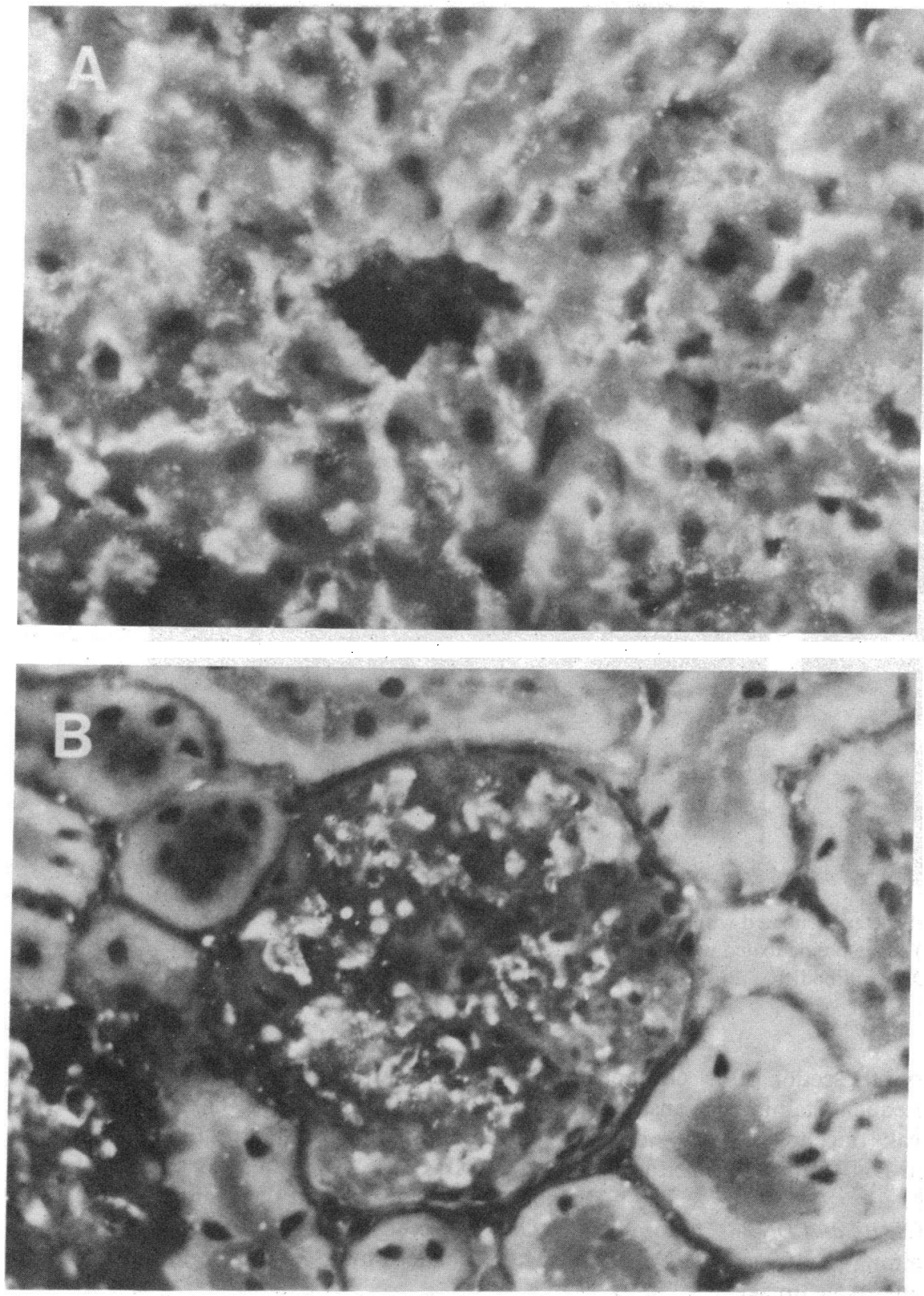

Figure 6. IF microscopy of liver $(A)$ and kidney $(B)$ tissues obtained $60 \mathrm{~min}$ after injection of DNP-GL $(5 \mathrm{mg} / \mathrm{kg})$. angial FN. In those experiments, DNP-GL was preincubated with purified $\mathrm{FN}$ at $37^{\circ} \mathrm{C}$ for $60 \mathrm{~min}$. The mixture was incubated with kidney slices, and after washing, the tissue was stained for DNP-GL (Fig. $7 C$ ). Preincubation with FN completely inhibited the binding of DNP-GL to the glomerular mesangium. However, these tissues demonstrated intense staining of all collagenous portions of the kidney, including Bowman's capsule and interstitium. Finally, preincubation of renal sections with rabbit anti-FN antibodies inhibited the binding of DNP-GL to the glomeruli (Fig. $7 \mathrm{D}$ ). Thus, purified FN and anti-FN antibodies inhibit the binding of DNP-GL to the glomerular mesangium, suggesting that DNP-GL binds to glomerular FN. Staining of collagenous structures by DNPGL-FN complexes is most likely due to binding of the FN portion of these complexes to collagen.

Characterization of DNP-GL IC and DNP-BSA IC. IC were formed with the antigens DNP-BSA and DNP-GL and a ra- diolabeled mouse monoclonal IgGl anti-DNP antibody. The same ratios of antigen to antibody $(1: 1, \mathrm{wt} / \mathrm{wt})$ were used for both IC preparations. After centrifugation at $1,000 \mathrm{~g}$ for 10 min, soluble IC were fractionated in sucrose gradients (Fig. 8 $A$ ). Both IC demonstrated a peak of radioactivity with a sedimentation coefficient of 8.5-10 S.

To test the ability of those IC preparations to bind to soluble FN, IC were incubated with EDTA anticoagulated plasma or purified $\mathrm{FN}$ for $15-60 \mathrm{~min}$ at $37^{\circ} \mathrm{C}$ and the mixture was fractionated in isokinetic sucrose gradients (Fig. $8 \mathrm{~B}$ ). There was no change in the size distribution of DNP-BSA IC. By contrast, DNP-GL IC preincubated with FN demonstrated a new subpopulation of IC with a sedimentation coefficient of $16 \mathrm{~S}$. This new subpopulation of heavier DNP-GL IC constituted $52 \%$ of the total IC preparation.

Incubation of DNP-GL IC with kidney slices indicated that, compared with DNP-GL alone, IC have a lower capacity 

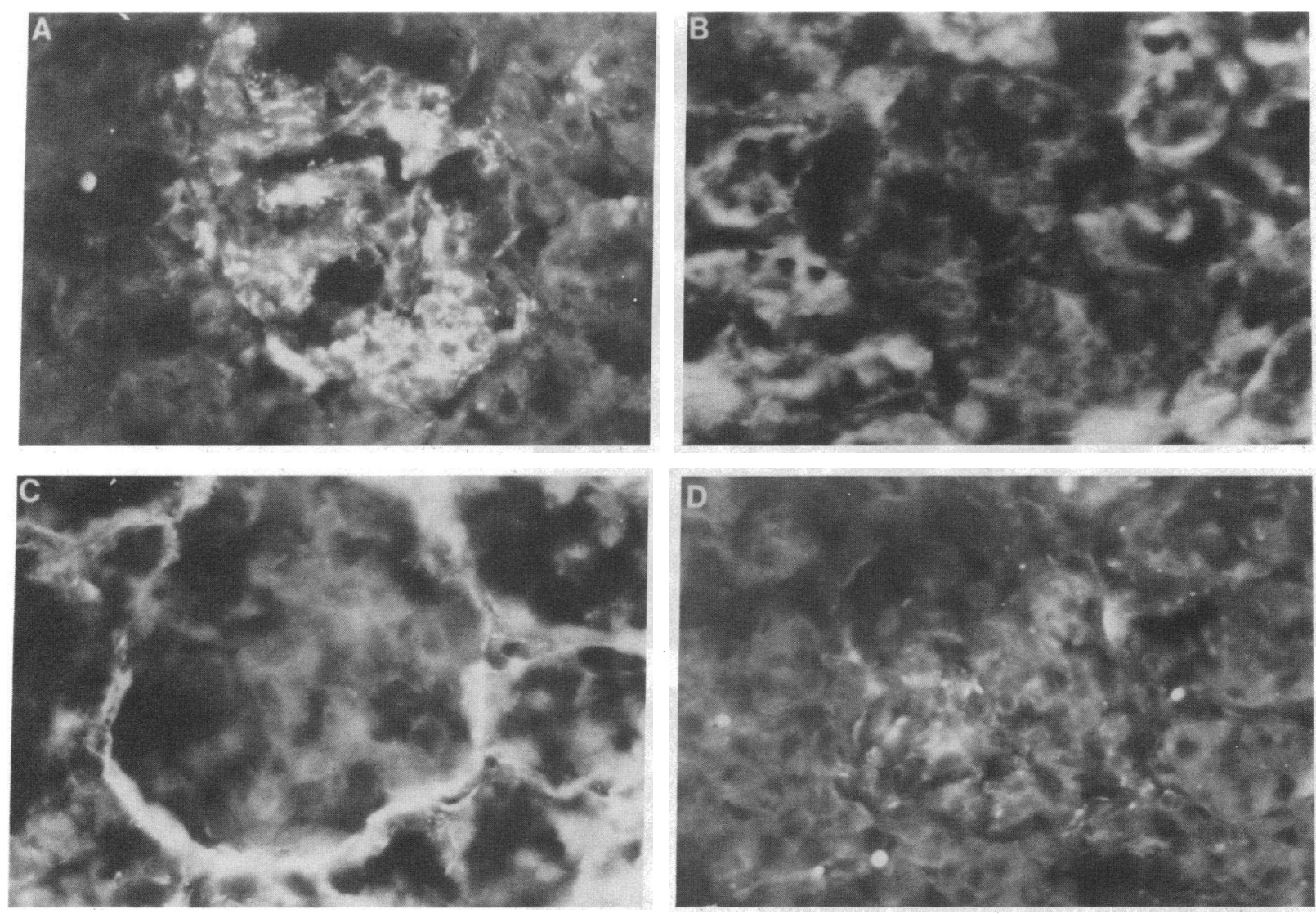

Figure 7. IF microscopy of cryostat sections of renal cortex incubated with $(A)$ DNP-GL, $(B)$ tissue preincubated with GL before DNP-GL, $(C)$ preformed complexes of DNP-GL and FN, and $(D)$ tissue preincubated with anti-FN antibodies before incubation with DNP-GL. All tissues were stained, by indirect IFluorescence, with anti-DNP antibodies.

to bind to tissue FN. Indeed, incubation of cryostat section of normal rat kidney with DNP-GL IC and subsequent staining for anti-DNP antibody demonstrated only weak (trace to $1+$ ) staining of the glomerular mesangium for anti-DNP antibodies, but stronger (2-3+) staining for DNP-GL.

Clearance and tissue deposition of DNP-GL IC and DNP$B S A I C$. Animals were injected with IC containing 50-100 $\mu \mathrm{g}$ of antigen. The removal rate, catabolic rate, and tissue uptake of IC was calculated as described above. Table III demonstrates the removal rate and tissue deposition of radiolabeled anti-DNP antibody alone and of DNP-GL IC and DNP-BSA IC. As can be seen, DNP-GL IC were removed from the circulation at a much faster rate than anti-DNP antibody alone or DNP-BSA IC. By ANOVA, there were significant differences in tissue deposition among groups (overall $P<0.0001$ ). Liver deposition of DNP-GL IC was higher than that of anti-DNP antibody alone or DNP-BSA IC. Renal deposition of DNP-GL IC was higher than the renal deposition of DNP-BSA IC, but lower than the renal deposition of DNP-GL alone (see Table I).

To further characterize the clearance mechanism of DNPGL IC, the removal from the circulation of IC was evaluated in animals pretreated with GL (an inhibitor of the clearance of DNP-GL) or heat aggregated IgG (AIgG) (an inhibitor of Fc receptor-mediated clearance) (Table IV). GL (10 mg/kg) caused a significant decrease in the removal rate of DNP-GL IC, indicating that the antigen DNP-GL participates in the clearance of DNP-GL IC. Also, GL-pretreated animals demonstrated lower splenic and renal deposition of DNP-GL IC. By contrast, AIgG did not cause a significant decline in the clearance rate of DNP-GL IC, suggesting that hepatic Fc receptors do not play a major role in the removal of these IC from the circulation. Indeed, the hepatic and splenic uptake of DNP-GL IC were enhanced by AIgG pretreatment.

\section{Discussion}

In the present study, we have shown that the ability of antigen or IC to bind to FN markedly influences the clearance and tissue deposition of that antigen or IC. Specifically, we have demonstrated that as early as 5 min after injection, DNP-GL binds to plasma proteins. The size similarity between DNPGL bound to plasma proteins in vivo and DNP-GL bound to purified FN in vitro, strongly suggests that, in vivo, DNP-GL binds to plasma FN. We also demonstrated that complexes of DNP-GL and FN are removed from the circulation faster than uncomplexed DNP-GL.

The liver is the major organ involved in the removal of DNP-GL from the circulation. Hepatic uptake of DNP-GL is specific for GL and is saturable. Based on previous studies, it can be postulated that the uptake of DNP-GL by the liver may be mediated by at least two mechanisms. First, complexes of DNP-GL and FN form in the circulation and bind directly to FN receptors on Kupffer cells $(12,13)$. Second, DNP-GL 

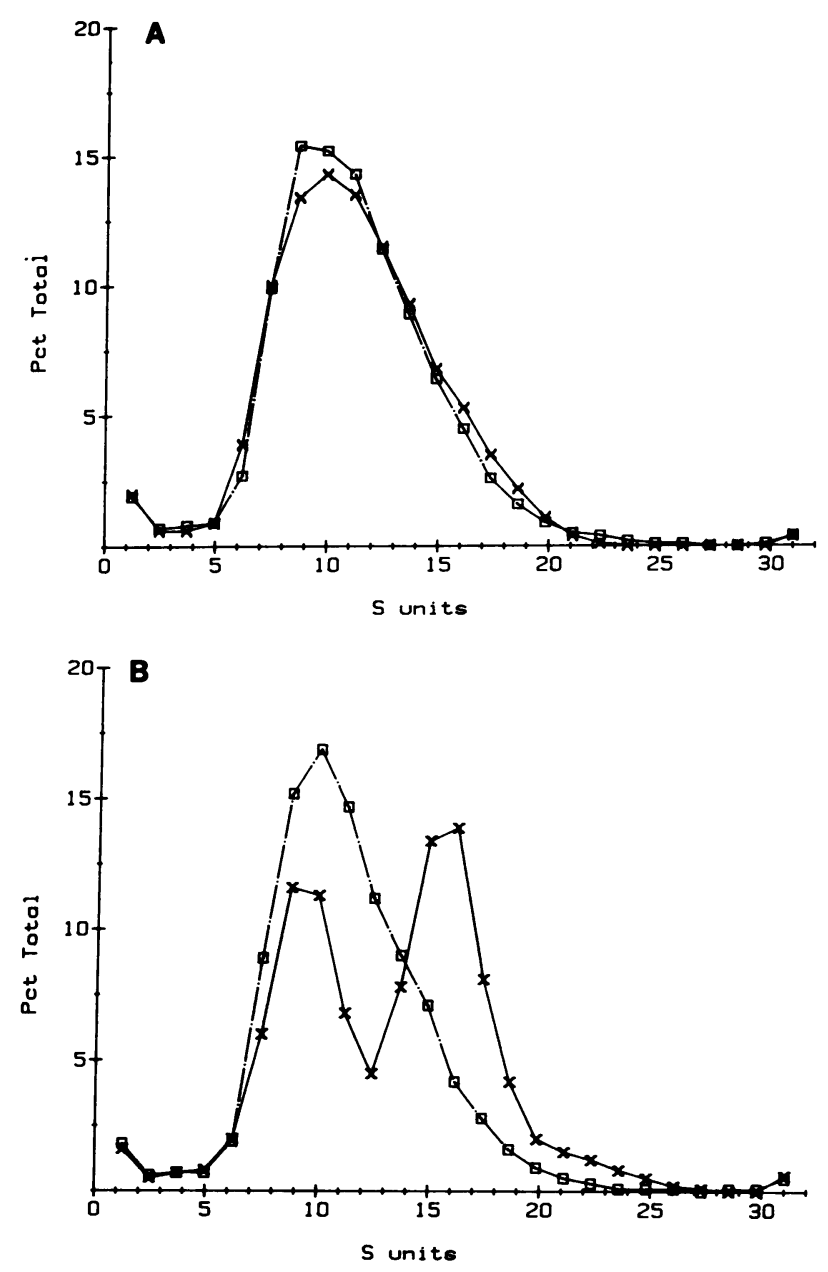

Figure 8. $(A)$ Isokinetic sucrose-gradient fractionation of radiolabeled DNP-BSA IC ( $\square$ ) and DNP-GL IC $(\times)$. (B) Isokinetic sucrose-gradient fractionation of radiolabeled DNP-BSA IC ( $\square$ ) and DNP-GL IC $(x)$ preincubated for 15 min with purified FN

binds directly to liver FN (24). The fact that the liver uptake of DNP-GL is saturable is compatible with a receptor-mediated uptake of the probe. Also compatible with the concept of a direct hepatic macrophage uptake of DNP-GL is the finding that DNP-GL was rapidly catabolized. Nonetheless, the above observations do not rule out the possibility that the initial uptake of DNP-GL by the liver is in part mediated by direct binding of the probe to tissue FN with a secondary transfer to the macrophage. Splenic uptake of DNP-GL, although lower per gram of tissue than hepatic uptake, is also specific for GL and saturable.
The data provided by the present studies taken together with previously reported in vitro data $(12,13,16)$ suggest the following hypothesis to explain the fate of a FN binding antigen in the circulation: once a FN binding antigen, such as DNP-GL, enters the circulation, it is rapidly bound by plasma FN. Complexes of DNP-GL and FN bind to specific FN receptors in hepatic Kupffer cells and splenic macrophages, and as a result, DNP-GL is phagocytozed and catabolized. This interpretation of the present findings is consistent with previous findings on the removal from the circulation of fibrogen degradation products (which have the capacity to bind to FN) (17) and with findings in patients with major body burns who demonstrate complexes of $\mathrm{FN}$ with degraded collagen in the circulation (18).

The renal uptake of DNP-GL was remarkably high. Indeed, at low doses of injected probe the renal and hepatic uptake of DNP-GL per gram of tissue was similar. However, at higher doses of DNP-GL, renal deposition of DNP-GL increased in direct proportion to the dose, whereas hepatic deposition was saturable. Thus, at higher doses of injected DNPGL, renal DNP-GL uptake per gram of tissue actually exceeded hepatic uptake. In in vitro studies we demonstrated that DNP-GL binds directly to renal FN and that the binding is saturable, but with concentrations of GL much higher than those achieved in vivo. We believe, based on the present finding, that the high renal uptake of DNP-GL is due to direct interaction of DNP-GL with renal FN. The presence of high concentration of $\mathrm{FN}$ in the glomerular mesangium (11) can explain the preferential deposition of DNP-GL in that location. The finding that, in vitro, preformed complexes of DNPGL and FN do not bind to mesangial FN suggests that plasma FN is an inhibitor of kidney deposition of antigens with FN binding capacity since, first, the formation of antigen-FN complexes accelerates the removal of the antigen from the circulation and, second, antigen-FN complexes do not bind to glomerular FN.

The ability of antigen to bind to FN also influenced the clearance and tissue uptake of IC formed with that antigen. This was shown by a comparison between DNP-BSA IC and DNP-GL IC. The following differences were demonstrated: $(a)$ DNP-GL IC bind to FN in vitro whereas DNP-BSA IC do not; (b) DNP-GL IC are removed from the circulation faster than DNP-BSA IC of similar size; $(c)$ hepatic and splenic uptake of DNP-GL IC was higher than that of DNP-BSA IC; and $(d)$ renal uptake of DNP-GL IC was significantly higher than that of DNP-BSA IC. These observations suggested that the removal from the circulation and tissue uptake of DNP-GL IC may be affected by the antigen since we have demonstrated that DNP-GL is cleared from the circulation rapidly. Indeed,

Table III. Removal Rate and Tissue Deposition, 60 Min after Injection, of Radiolabeled Anti-DNP Antibodies, DNP-BSA IC, and DNP-GL IC

\begin{tabular}{|c|c|c|c|c|c|c|}
\hline Probe & $N$ & Removal rate & Liver & Spleen & Kidney & Lung \\
\hline & & $\% I D / 30 \mathrm{~min}$ & & & & \\
\hline Anti-DNP & 2 & $2 \pm 0.5$ & $\mathrm{ND}^{*}$ & ND & ND & ND \\
\hline DNP-BSA IC & 2 & $18 \pm 1.5$ & $3.5 \pm 0.8$ & ND & ND & ND \\
\hline DNP-GL IC & 5 & $76 \pm 1.2$ & $8.3 \pm 2$ & $0.3 \pm 0.02$ & $0.22 \pm 0.02$ & $0.15 \pm 0.05$ \\
\hline
\end{tabular}

Tissue counts were corrected for blood contamination. * ND, not detected. 
Table IV. Removal Rate and Tissue Deposition of DNP-GL IC in Rats Pretreated with GL (10 mg/kg) or AIgG (30 mg)

\begin{tabular}{lllllll}
\hline Inhibitor & $N$ & Removal rate & Liver & Spleen & Kidney & Lung \\
\hline & & $\% I D / 30 \mathrm{~min}$ & & & & \\
GL & 3 & $62 \pm 3.8^{*}$ & $6.4 \pm 1.4^{\ddagger}$ & $0.08 \pm 0.08$ & $0.07 \pm 0.07$ & ND \\
AIgG & 3 & $70 \pm 4.6$ & $10.4 \pm 1$ & $0.61 \pm 0.1$ & ND & \\
\hline
\end{tabular}

* Removal rates were compared with the removal rate of DNP-GL IC in the absence of inhibitors by ANOVA with Newman-Keuls multiple comparison test. ${ }^{\ddagger}$ Tissue deposition was compared to that of DNP-GL IC, without inhibition, by ANOVA with repeated measures. ${ }^{8}$ ND, not detectable.

GL (a specific inhibitor of DNP-GL clearance) causes a significant inhibition of the removal rate and splenic uptake of DNP-GL IC. Hepatic uptake of DNP-GL IC was lowered, but not significantly, by GL pretreatment. It may be that splenic uptake of DNP-GL IC is more sensitive to GL inhibition than hepatic uptake of that probe. However, it may be also that the lack of significant effect of GL on the hepatic uptake of DNPGL IC is due to large animal-to-animal variations, especially among animals not pretreated with GL (from 4.4 to $13.2 \%$ ID/g). Also, we have shown that AIgG, a known inhibitor of Fc receptor-mediated clearance, has no significant effects on the clearance of DNP-GL IC. Furthermore, AIgG enhanced hepatic and splenic uptake of DNP-GL IC, an effect previously observed with other IC systems (25). These experiments indicated that $\mathrm{Fc}$ receptors have no evident role on the removal of DNP-GL IC from the circulation. The similarities between the present observations and previous studies on the removal from the circulation of DNA IC are striking. Emlen and Mannik (26) have suggested that DNA plays a role on the clearance of DNA IC from the circulation. Also, recent evidence indicates that DNA IC are not removed from the circulation by Fc receptor-mediated mechanisms (27). Other studies have indicated that certain antigens play a major role on the clearance from the circulation of IC formed with that antigen (28).

Renal uptake of DNP-GL IC was significantly higher than that of DNP-BSA IC or anti-DNP antibody alone. However, the renal uptake of DNP-GL IC was only a fraction of the renal deposition of DNP-GL alone. Consistent with this in vivo observation, in vitro we demonstrated that DNP-GL IC have a much lower affinity for renal tissues than DNP-GL. This observation is in contrast with the binding of DNP-GL IC to soluble FN. Thus, it appears that anti-DNP antibodies inhibit the binding of DNP-GL to tissue FN thus preventing, at least in part, renal deposition of these IC.

A FN-specific mechanism of removal from the circulation and tissue deposition of antigens and IC could have important implications in our understanding of IC-mediated diseases in humans in which FN binding antigens such as DNA and bacterial antigens may be involved.

\section{Acknowledgments}

We thank Dr. L. A. Hebert for his valuable advice in the preparation of this manuscript. We thank Dr. Sedmak (Pathology Department, Ohio State University) for his assistance in the preparation and interpretation of microscopic slides. We thank Mrs. Dorothy Walker and Mrs. Carmela Price for their expert secretarial assistance.

This work was supported by grants from The National Kidney Foundation of Central Ohio and by a seed grant from The Ohio State University.

\section{References}

1. Couser, W. G., and D. J. Salant. 1980. In situ immune complex formation and glomerular injury. Kidney Int. 17:1-13.

2. Mannik, M., W. P. Arend, A. P. Hall, and B. C. Gilliland. 1971. Studies on antigen-antibody complexes. I. Elimination of soluble complexes from rabbit circulation. J. Exp. Med. 133:713-739.

3. Mannik, M., L. Y. C. Agodoa, and K. A. David. 1983. Rearrangement of immune complexes in glomeruli leads to persistence and development of electron-dense deposits. J. Exp. Med. 157:1516-1527.

4. Adler, S. G., H. Wang, H. J. Ward, A. H. Cohen, and W. A. Border. 1983. Electrical charge. Its role in the pathogenesis and prevention of experimental membranous nephropathy in the rabbit. $J$. Clin. Invest. 71:487-499.

5. Golbus, S. M., and C. B. Wilson. 1979. Experimental glomerulonephritis induced by in situ formation of immune complexes in glomerular capillary wall. Kidney Int. 16:148-157.

6. Zardi, L., A. Siri, B. Carnemolla, and L. Santi. 1979. Fibronectin: a chromatin-associated protein? Cell. 18:649-657.

7. Proctor, R. A., D. F. Mosher, and P. J. Olbrantz. 1982. Fibronectin binding to staphylococcus aureus. J. Biol. Chem. 257:1478814794.

8. Myhre, E. B., and P. Kuusela. 1983. Binding of human fibronectin to group A, C, and G streptococci. Infect. Immun. 40:29-34.

9. Izui, S., P.-H. Lambert, and P. A. Miescher. 1976. In vitro demonstration of a particular affinity of glomerular basement membrane and collagen for DNA: a possible basis for a local formation of DNA-anti-DNA complexes in systemic lupus erythematosus. J. Exp. Med. 144:428-443.

10. Lake, R. A., A. Morgan, B. Henderson, and N. A. Staines. 1985. A key role for fibronectin in the sequential binding of native dsDNA and monoclononal anti-DNA antibodies to components of the extracellular matrix: its possible significance in glomerulonephritis. Immunology. 54:389-395.

11. Courtoy, P. J., Y. S. Kanwar, R. O. Hynes, and M. G. Farquhar. 1980. Fibronectin localization in the rat glomerulus. J. Cell. Biol. 87:691-696.

12. Bevilacqua, M. P., D. Amrani, M. W. Mosesson, and C. Bianco. 1981. Receptors for cold-insoluble globulin (plasma fibronectin) on human monocytes. J. Exp. Med. 153:42-60.

13. Pommier, C. G., J. O'Shea, T. Chused, K. Yancey, M. M. Frank, T. Takahashi, and E. J. Brown. 1984. Studies on the fibronectin receptors of human peripheral blood leukocytes: morphologic and functional characterization. J. Exp. Med. 159:137-151.

14. Pommier, C. G., S. Inada, L. F. Fries, T. Takahashi, M. M. Frank, and E. J. Brown. 1983. Plasma fibronectin enhances phagotyctosis of opsonized particles by human peripheral blood monocytes. $J$. Exp. Med. 157:1844-1854.

15. Cosio, F. G. 1984. Human fibronectin is an opsonin for IgG antibody-coated sheep erythrocytes. J. Lab. Clin. Med. 103:613-619.

16. Van de Water, L., III, S. Schroeder, E. B. Crenshaw III, and R. O. Hynes. 1981. Phagocytosis of gelatin-latex particles by a murine macrophage line is dependent on fibronectin and heparin. J. Cell. Biol. 90:32-39. 
17. Sherman, L. A., and J. Lee. 1982. Fibronectin: blood turnover in normal animals and during intravascular coagulation. Blood. 60:558-563.

18. Deno, D. C., M. H. McCafferty, T. M. Saba, and F. A. Blumenstock. 1984. Mechanism of acute depletion of plasma fibronectin following thermal injury in rats. Appearance of a gelatinlike ligand in plasma. J. Clin. Invest. 73:20-34.

19. Ruoslahti, E., and E. Engvall. 1978. Immunochemical and collagen-binding properties of fibronectin. Ann. NY Acad. Sci. 313:178-191.

20. Waxman, F. J., L. A. Hebert, F. G. Cosio, W. L. Smead, M. E. VanAman, J. M. Taguiam, and D. J. Birmingham. 1986. Differential binding of immunoglobulin $A$ and immunoglobulin $G 1$ immune complexes to primate erythrocytes in vivo. Immunoglobulin A immune complexes bind less well to erythrocytes and are preferentially deposited in glomeruli. J. Clin. Invest. 77:82-89.

21. Steinbuch, M., and R. Audran. 1969. The isolation of IgG from mammalian sera with the aid of caprylic acid. Arch. Biochem. Biophys. 134:279-284.

22. Johns, P., and D. R. Stanworth. 1976. A simple numerical method for the construction of isokinetic sucrose density gradients and their application to the characterization of immunoglobulin complexes. J. Immunol. Methods. 10:231-252.

23. Stewart, G. A., and P. Johns. 1976. Empirical and theoretical relationships between the sedimentation coefficient and molecular weight of various proteins, with particular reference to the immunoglobulins. J. Immunol. Methods. 10:219-229.

24. Martinez-Hernandez, A. 1984. The hepatic extracellular matrix. I. Electron immunohistochemical studies in normal rat liver. Lab. Invest. 51:57-74.

25. Jimenez, R. A. H., and M. Mannik. 1982. Evaluation of aggregated IgG in mice as an Fc receptor specific probe of the hepatic mononuclear phagocyte system. Clin. Exp. Immunol. 49:200-208.

26. Emlen, W., and M. Mannik. 1982. Clearance of circulating DNA-Anti-DNA immune complexes in mice. J. Exp. Med. 155:1210-1215.

27. Clarkson, S. B., R. P. Kimberly, J. E. Valinsky, M. D. Witmer, J. B. Bussel, R. L. Nachman, and J. C. Unkeless. 1986. Blockade of clearance of immune complexes by an anti-Fc receptor monoclonal antibody. J. Exp. Med. 164:474-500.

28. Finbloom, D. S., D. B. Magilavy, J. B. Harford, A. Rifai, and P. H. Plotz. 1981. Influence of antigen on immune complex behavior in mice. J. Clin. Invest. 68:214-224. 